\title{
The First End-to-End Live Trial of CBRS with Carrier Aggregation using 3.5 GHz LTE Equipment
}

\author{
Marko Palola and Pekka Aho \\ VTT Technical Research Centre of Finland \\ Marko Mäkeläinen, Kalle Lähetkangas, Satya Joshi \\ Centre for Wireless Communications, University of Oulu, \\ Finland
}

\author{
Tero Kippola \\ Centria University of Applied Sciences, Finland \\ Arto Kivinen and Juhani Hallio \\ Turku University of Applied Sciences, Finland
}

Vesa Hartikainen and Lucia Tudose

Nokia, Finland

\begin{abstract}
This demonstration presents the first end-to-end field trial of the US three-tier Citizens Broadband Radio Service (CBRS) with carrier aggregation using commercial 3.5 GHz network elements (UEs, eNBs, EPC) and platforms (NMS, SON). The live trial shows how shared spectrum is assigned to an operational LTE base station and how it boosts the end user mobile data rate due to extended bandwidth availability. Also, the performance of the CBRS is evaluated and compared to the FCC requirements for the evacuation time.
\end{abstract}

Keywords-dynamic spectrum sharing; spectrum access system; citizens broadband radio service;

\section{INTRODUCTION}

A field trial of the US three-tier Citizens Broadband Radio Service (CBRS) is presented to provide higher bit rates for mobile broadband users by operating carrier aggregation on shared spectrum. A mobile network operator (MNO) has licensed $10 \mathrm{MHz}$ spectrum from 3GPP band 42 for LTE and when granted by Spectrum Access System (SAS), the operator boosts the existing broadband service by using shared spectrum over 3GPP band 48 for CBRS.

Carrier aggregation is a technology in LTE-Advanced (3GPP Release 10 and above) that allows combining separate spectrum bands as one and increase the bandwidth available to LTE base stations, aka. eNBs. Combined bandwidth shows improved mobile data throughput to end users.

The presented field trial is part of the Finnish spectrum sharing trial continuum that began in 2013 with the World's First Licensed Shared Access (LSA) trials [1,2] and evolved to SAS/CBRS field trial in 2016 [3].

\section{The ARChiteCtURE OF FIELD TRIAL}

In CBRS the spectrum sharing is managed by SAS. In this demonstration we present SAS elements including Domain Proxy, Environmental Sensing Capability (ESC), SAS server, and SAS algorithms, developed by research partners in CORE++ project [4], following the Federal Communications Commission's (FCC) definitions and Wireless Innovation Forum (WInnF) Spectrum Sharing Committee's recommendations [5]. The field trial is built on commercial LTE network elements: user equipments (UEs), eNBs, and evolved packet core (EPC), and platforms: network management system (NMS) and Self-Organizing Networks (SON). The architecture of this field trial is shown in Figure 1.

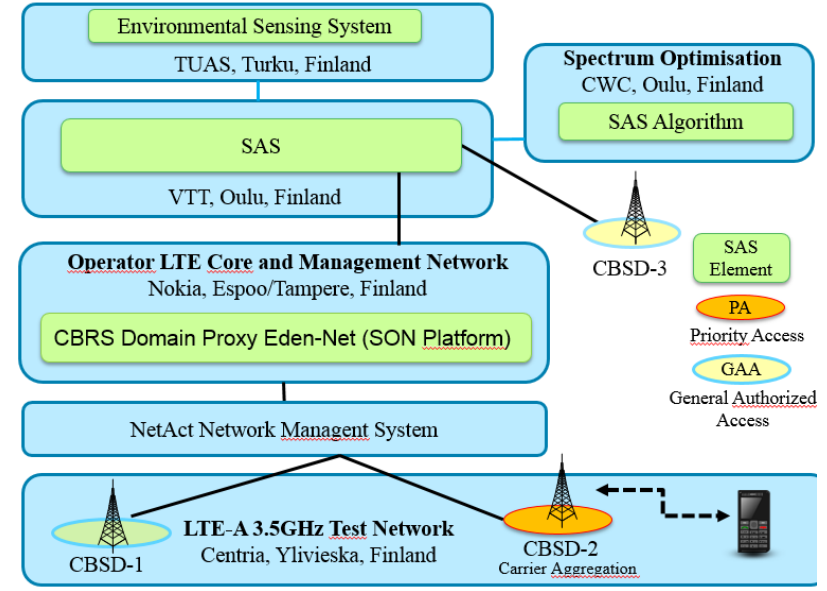

Fig 1. The architecture of the field trial. The base station CBSD-2 is used for demonstrating the benefits of CBRS with carrier aggregation.

In the field trial, a radar signal simulator system was used to generate a real signal according to SPN-43 radar characteristics. Radar sensing system consist of spectrum sensor and ESC software. Spectrum is sensed and power level values are recorded in the CBRS band. Occupancy scan is repeated over every 45 seconds. The developed ESC software processes the occupancy data and detects narrow band power levels higher than $-90 \mathrm{dBm}$ in the CBRS band. The detected radar presence is informed to SAS.

An advanced SAS Algorithm has been developed for managing interference and distributing the shared spectrum optimally to base stations. The SAS algorithm protects the Incumbent and PALs from interference while it maximizes the distance of base stations on same channel and balances 
the number of base stations in each channel. In the trial shared spectrum is managed using four $10 \mathrm{MHz}$ channels and having nine census tracts at Ylivieska town, so each census tract have $40 \mathrm{MHz}$ of shared band. Three of the base stations are real commercial $3.5 \mathrm{GHz}$ eNBs and the rest of them in adjacent census tracts are simulated.

Domain Proxy (DP) is implemented as a SON module into network management system (NMS). NMS configures the eNBs based on commands issued by the DP module. In this demonstration, we have three live $3.5 \mathrm{GHz}$ eNBs: CBSD-1, CBSD-2, and CBSD-3. The carrier aggregation (CA) capabilities are demonstrated with CBSD-2 using the shared spectrum as additional bandwidth for ongoing operation, and the throughput of the end-user is measured with customer-premises equipment (CPE). In practice, the licensed spectrum is configured to the eNBs as one cell and the aggregated band as another cell. This allows both cells to be configured separately. CA-enabled customer equipment also combines the two associated CA cells and operates them at the same time.

\section{CBRS TEST NETWORK AND END USER EQUIPMENT}

The CBRS test network consist of commercially available LTE-Advanced compliant eNBs, a radio access network and a core network. eNBs are commercial Flexi multiradio time-division (TD)-LTE $3.5 \mathrm{GHz}$ operating with a test licence from FICORA for 3510 - $3590 \mathrm{MHz}$.

The end user device used is 3GPP Rel.10/11 compliant LTE category 6 single-mode indoor customer premises equipment (CPE). It is an LTE-router that uses LTE connection for the WAN connection and routes the connection via various connection methods, such as LAN or WLAN. CPE can support 2 Component Carrier (CC) intraband carrier aggregation up to $40 \mathrm{MHz}$. Also it supports both contiguous and non-contiguous component carrier aggregation.

\section{DEMONSTRATION}

The carrier aggregation field trial is demonstrated live, but the LTE network, ESC, and SAS are located in Finland. In the demonstration, we will show three live views to the remote field trial: 1) Radar signal remote control, 2) graphical visualisation of the system including the throughput of the CPE, and 3) Domain Proxy view for the operator. We will show how the cell providing carrier aggregation is enabled and how the bitrate of the mobile user is increased when additional bandwidth is is allocated for CA by the SAS.

The graphical visualization has several views to monitor the trial network in real-time: topology view, state view, and performance analysis tool view. In the topology view, the allocated bands for each trial network entity are show on the channel allocation table, providing a convenient manner to understand spectrum usage of the trial network area. The state view shows the current state of each CBSD from the SAS controller point-of-view. Third view of the visualization is the performance analysis tool view providing an easyreadable monitor panel to follow the performance of the system. Also, it illustrates the carrier aggregations occurring in the trial network. Figure 2 illustrates the topology, state, performance analysis tool views.

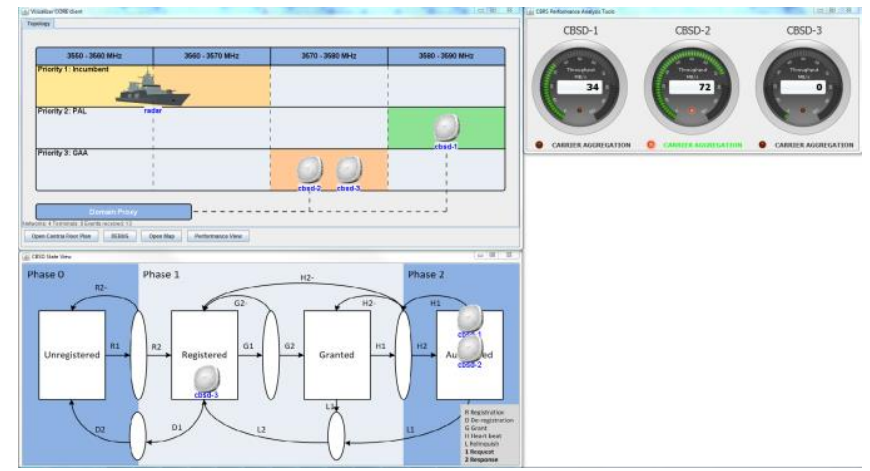

Fig 2. The graphical visualization views of the demonstration.

\section{ACKNOWLEDGMENTS}

Field trial is done in the CORE++ research project [4] within the 5thGear program of Tekes - the Finnish Funding Agency for Technology and Innovation. The authors would like to acknowledge the CORE++ project consortium: VTT Technical Research Centre of Finland, University of Oulu, Centria University of Applied Sciences, Fairspectrum, Turku University of Applied Sciences, Nokia, PehuTec, Bittium, KeySight, Finnish Defence Forces, Finnish Communications Regulatory Authority and Tekes.

\section{REFERENCES}

[1] M. Matinmikko, M. Palola, H. Saarnisaari, M. Heikkila, J. Prokkola, T. Kippola, T. Hanninen, M. Jokinen, S. Yrjola, Cognitive radio trial environment: First live authorized shared access-based spectrumsharing demonstration, Vehicular Technology Magazine IEEE, vol. 8, no. 3, pp. 30-37, Sept 2013.

[2] M. Palola et al., "Live field trial of licensed shared access (LSA) concept using LTE network in $2.3 \mathrm{GHz}$ band," in Proc. DySPAN, pp. 38-47, Apr. 2014.

[3] P. Aho et al., "Field Trial of Citizens Broadband Radio Service (CBRS) / Spectrum Access System (SAS), "Wireless Innovation Forum European Conference on Communications Technology and Software Defined Radio (WInnComm-Europe), Paris, France Oct. 2016

[4] The CORE++ project web site. [Online]. Available: http://core.willab.fi. [Accessed: 16-Dec-2016].

[5] Technical Specification Document WINNF-16-S-0016 Version V1.0.0, 29 November 2016. 